\title{
Application of Fractal Analysis Methods of Textures Earth Surface Images for Ecological Setting Assessment
}

\author{
Nikolay Akinshin ${ }^{1}$, Oleg Esikov ${ }^{1}$, Alexander Potapov ${ }^{1,2 *}$, Ruslan Akinshin ${ }^{1}$, and Alexander Kuleshov ${ }^{1}$ \\ ${ }^{1}$ Kotelnikov Institute of Radioengineering and Electronics, Russian Academy of Sciences, RU-125009, Moscow, Russia \\ ${ }^{2}$ E JNU-IREE Joint Laboratory of Fractal Method \& Signal Processing, Department of Electronic Engineering, College of Information \\ Science and Technology, JiNan University, Guangzhou, China
}

\begin{abstract}
The use of fractal analysis methods for automatic textures earth surface image evaluation and ecological setting assessment has been proposed. Image processing techniques have been chosen for the following fractal analysis. The use of the Minkowski dimension to construct sheet dimension and histogram of fractal dimension has been suggested. Performance tests for the proposed methods and algorithms have been performed and analysed presented. Application of the fractal analysis methods is efficient in solving problems of monitoring objects clustering, assessing their conditions, identifying anomalies that have implications for the ecological setting, and evaluating the development of anomalous situations. The fractal analysis algorithms permit efficient processing of array information in the case, when they are implemented on modern computational hardware platforms.
\end{abstract}

\section{Introduction}

Modern means of earth remote sensing (ERS) include systems placed on the board of aerospace vehicles and unmanned aerial vehicles.

Among the many implemented and attractive projects one should highlight [1]: foreign space-based systems "ERS-2" and "Envisat" (Europe)," Radarsat-2" (Canada), "ALOS” and "IGS” (Japan), "TerraSAR-X” (Germany), "CosmoSkyMed” (Italy), "RSS / JB” (China), russian "Condor", "Resurs-DKR", "Smotr", "Sever”, "Monitor”, “Arkon”; air "Hisar” (USA), "ESAR” (Germany), "EL / M-2060P SAR” (Israel), "Imark" and "Compact" (Russia); funds on unmanned vehicles "AN / ZPQ-1 TESAR" and "AN / APY-8 Lynx SAR / GMTI" (USA), "EL / M-2055 SAR / MTI" (Israel).

The instruments and systems in question solve the following tasks to the extent of ecological setting monitoring [1, 2]:

1. Monitoring of natural disasters areas.

2. Monitoring of forestland and water files, agricultural land condition and so on.

3. Monitoring and traffic conditions setting, etc.

The images received by these means are distinguished by potentially high resolving power, so they are a key element of modern and advanced data systems.

Algorithms and methods of image fractal analysis are one of the most advanced ways of images processing transferred from the means of earth remote sensing and monitoring [3-5].

\section{Formulation of the problem}

A feature of fractal methods is that, when terrain images processing, it is possible to describe the uniqueness of the observed object using one parameter - the fractal dimension, which gives the opportunity to carry out a preliminary automatic segmentation of the analyzed image by selecting clusters of a given range of fractal dimensions. By the process of fractal processing is meant the sequential operation of the following phases:

1. A field (sheet) construction of fractal image dimension and its thresholding.

2. Histograming of fractal image dimension and its analyzing.

3. Image clustering based on the field of fractal dimension.

A histogram of the fractal dimension levels distribution, which has borders in a certain range, is one of the options for presenting the results of fractal image analysis.

It is possible to organize an automated classification of objects on the analysed image and their clustering based on the obtained histogram.

There are good reasons to use the isarhythm method $[3,4]$, based on contour lines of objects and surfaces usage with the application of dimension reduction technique to improve the quality of the result obtained.

The following 3D methods of preprocessing were used to prepare images for the construction of fractal dimension sheet: histogram equalization, Sobel transformation, Kirsch transformation, Laplace transformation, threshold transformation [4]. 
Its black and white version is the result of image processing, on the basis of which the sheet and histogram of the fractal dimension are calculated.

\section{Solution methods}

Image points are subjected to threshold processing relative to the selected section level in intensity In. If $I(i$, $j)>I_{n}$ then the point is set to one, if $I(i, j)<I_{n}$, then to zero.

The construction of the fractal image dimension sheet was carried out in a sliding display field of size $K \times K$ points with a given period $s=K$. To calculate the fractal dimension of image fragment bounded by the display field, the quadrate method was used with the help of Minkowski dimension [3, 4]. The level of image area fractal dimension $D$ in the display field depends on its intention, as well as the size of the display field, and is in the interval $[0,2.0]$. As the display field size is zooming, the fractal dimension level of the corresponding image fragment is growing to a large size.

Analysis of the fractal dimension histogram allows to select the features of the image objects and to perform clustering, and assessment of occupied display area fraction.

\section{Numerical experimental results}

In Figures 1-4 show the results of fractal image analysis, which correspond to tasks 1 and 2, solved during earth remote sensing.

In these figures you can see that the application of image preprocessing methods allows to construct a fractal image dimension sheet using the Minkowski dimension.

In Fig. 1 shows an image fragment containing a forest, a reservoir, and a system of natural soil roads. The Sobel transformation was applied in the image preprocessing.

In addition, the histogram of the fractal dimension has 3 obvious bursts over the intervals of $0-0.1$; $0.6-1.0$; 1.4-1.65.

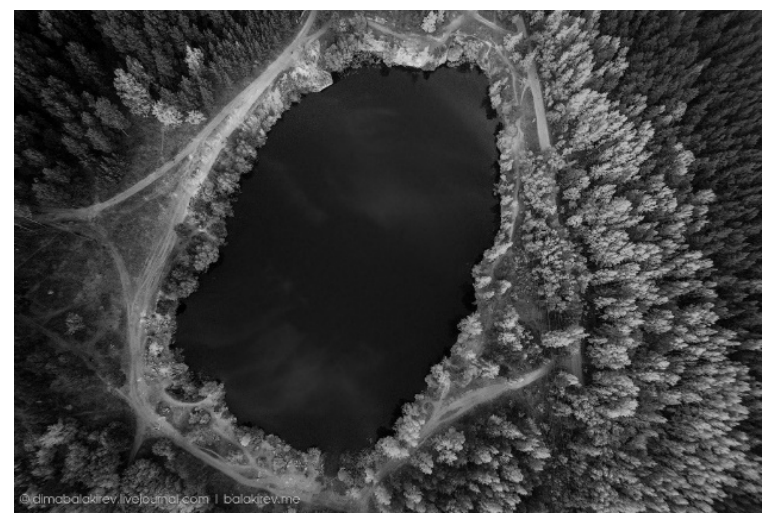

(a)

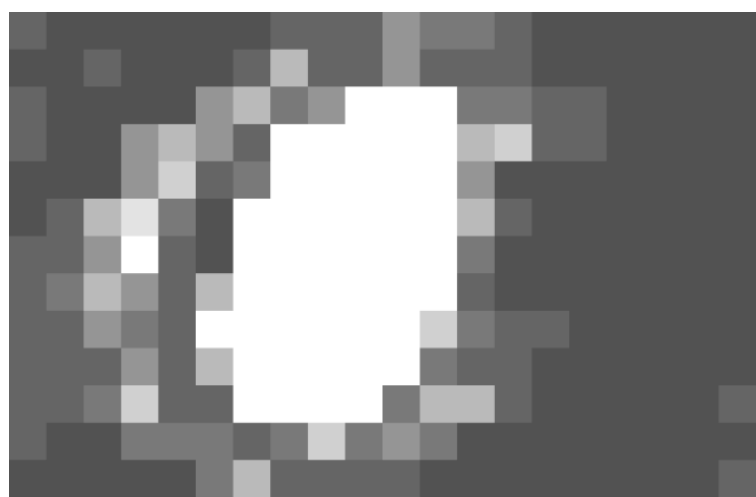

(b)

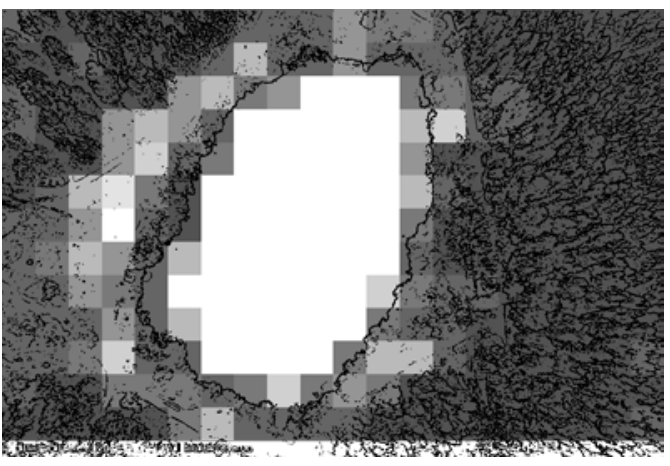

(c)

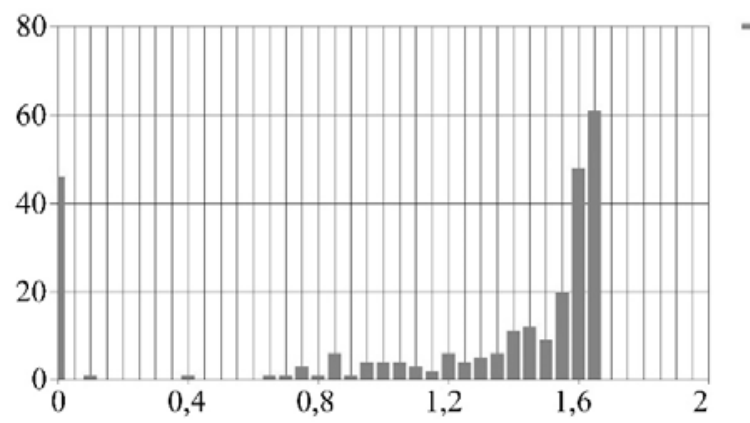

(d)

Fig. 1. The results of fractal image analysis (image size $800 \times 541$; fractal dimension $D$ estimation display field size 40): (a) - the original image; (b) - fractal dimension sheet; (c) combined image; (d) - histogram of the fractal image dimension.

Analysis of the received fractal dimension histogram (Fig. 1d) made it possible to estimate the display area fraction occupied by objects such as forest and a reservoir in the image. So, for the image shown in Figure 1 , the forest fraction is $52 \%$, and the area occupied by the lake is $21 \%$ of the total image area for which the histogram is constructed.

In Fig. 2 is represented a wood with an island of fire with a corresponding sheet and a fractal dimension histogram.

The fractal dimension $D$ of the image areas corresponding to the solid wood is in the range of 1.501.65. Fig. 2c and Fig. 2d show fractal dimension histograms of the naive wood and the same wood with a fire. The occurence of smoke as a result of a forest fire reduces the contrast and texture of the corresponding image areas, which leads to the appearance of areas 
corresponding fractal dimensions in the interval from 0 to 1.15 on the histogram, which are no in the histogram of the original wood image.

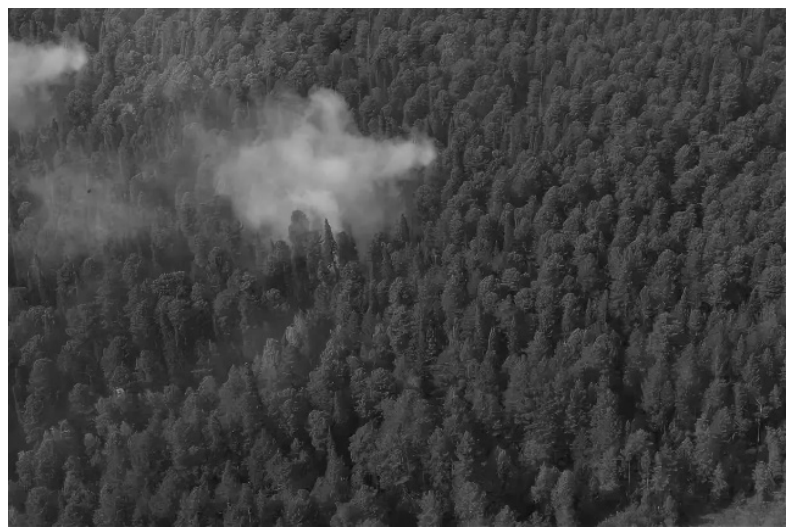

(a)

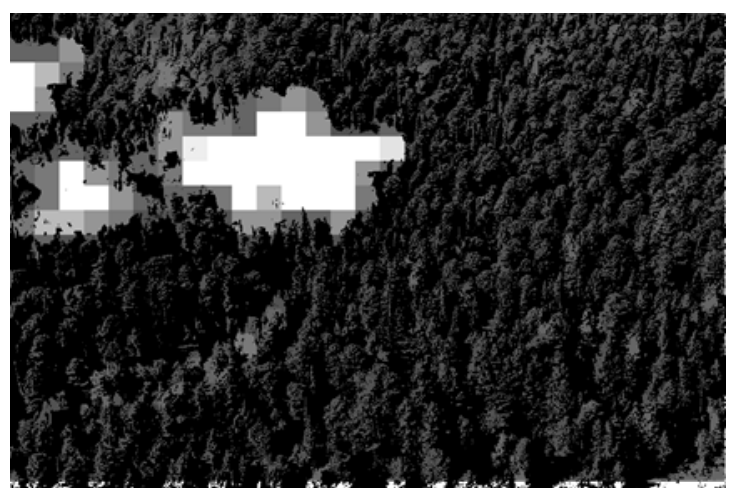

(b)

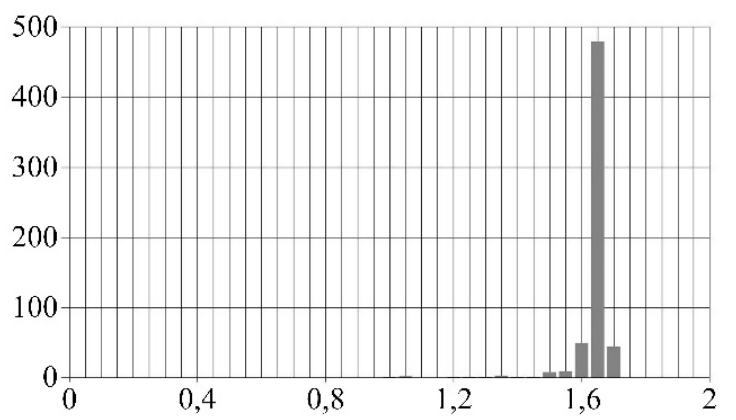

(c)

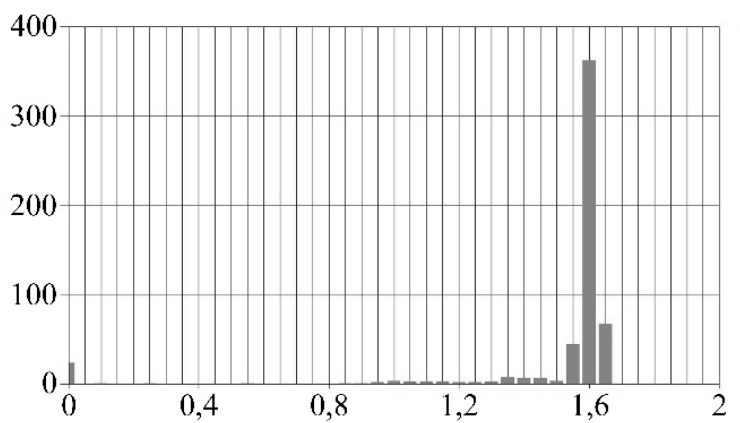

(d)

Fig. 2. The results of fractal image analysis (image size $800 \times 541$; fractal dimension $D$ estimation display field size 40): (a) the original image; (b) - combined image; (c) histogram of the fractal image dimension of the original wood; (d) - histogram of the fractal wood image dimension with fire.
The corresponding fragment of the fractal dimension histogram (Fig. 2d) in the interval from 0 to 1.2 is shown in Fig. 3.

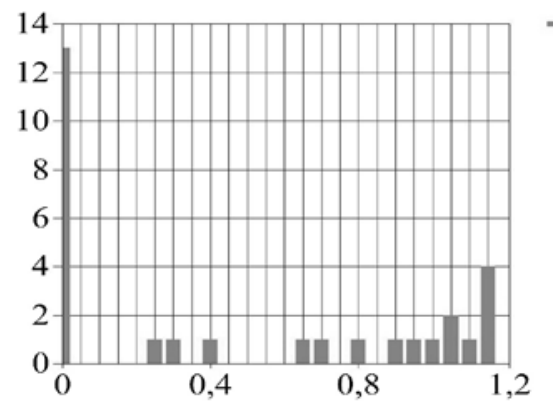

Fig. 3. The fragment of a fractal dimension histogram corresponding to the centers of a forest fire.

Histogram analysis (Fig. 2d) allows to conclude that there is a fire hazard and to make a raw estimation of its location (according to screening with smoke zone) and area (about $5 \%$ of the image area).

Fig. 4 shows a fragment of an image containing a section of the sea surface with an oil spill.

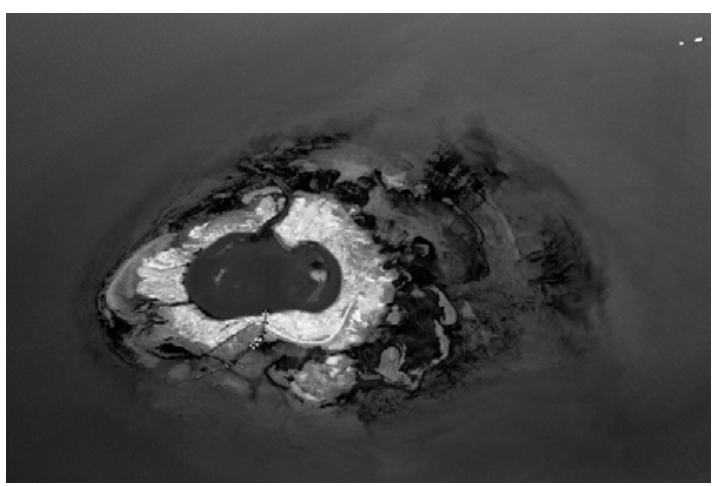

(a)

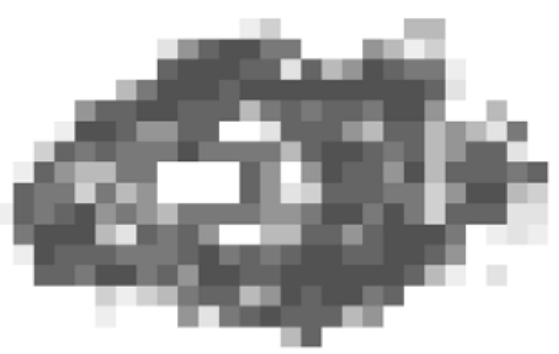

(b)

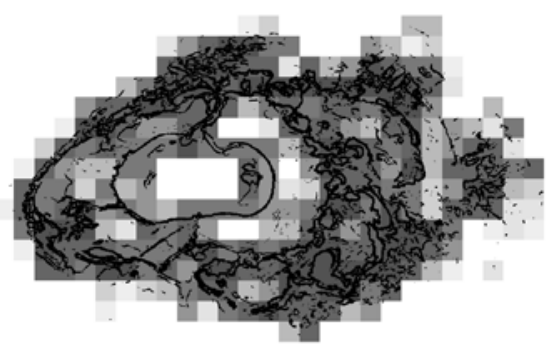

(c) 


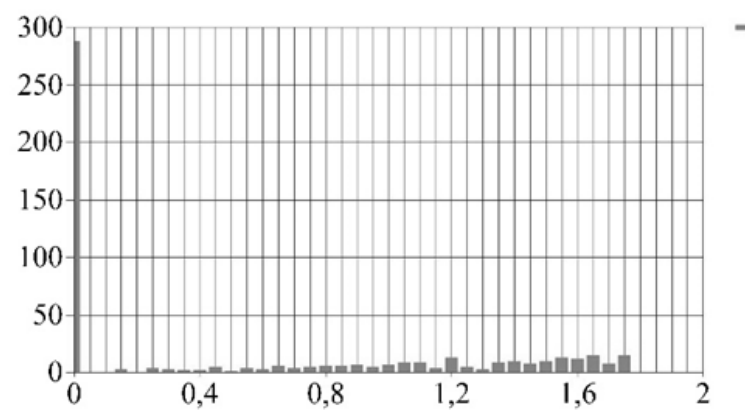

(d)

Fig. 4. The results of a fractal analysis of the sea surface section image with an oil spill (image size $1124 \times 744$; fractal dimension $D$ estimation display field size - 30): (a) - original image; (b) - fractal dimension sheet; (c) - combined image; (d) - histogram of the fractal image dimension.

The Sobel transformation was applied in the image pre-processing. Due to the fact that free of oil sea surface in the figure does not have a evident sign of structure, after applying the Sobel transformation the fractal dimension of such image areas will be close to 0 , which you can distinctly observe on the histogram (Fig. 4d). The areas of the image corresponding to the areas of the sea surface that have been contaminated have a fractal dimension other than zero. The area of pollution corresponds to $47 \%$ of the total image area. It should be noted that the use of image transformations with the first-order and second derivatives (Kirsch, Sobel, Laplace, etc.) do not allow to identify the zones of greatest contamination that have a homogeneous structure.

\section{Conclusion}

Thus, the application of the fractal analysis methods allows solving successfully the problems of monitoring objects clustering, to assess their condition, to identify anomalies that have implications for the ecological setting, as well as to evaluate the development of anomalous situations. The computational complexity of the fractal analysis algorithms permits efficiently to process a great deal of array information in the case of their implementation on modern computing hardware.

\section{References}

1. R.A. Schowengerdt, Remote sensing: models and methods for image processing (Academic Press, Burlington, 2007)

2. A. S. Danilov, Ur. D. Smirnov, M. A. Pashkevich, Russian Journal of Ecology, 46 (1), 14-19 (2015)

3. A. A. Potapov, Fractals in Radiophysics and Radar: Sampling Topology (Universitetskaya Kniga, Moscow, 2005)

4. A. A. Potapov, Yu. V. Gulyaev, S. A. Nikitov et al., The Latest Techniques of Image Processing (Fizmatlit, Moscow, 2008)
5. Yu. V. Gulyaev, A. A. Potapov, J. Communications Technology and Electronics, 64(9), 911-925 (2019) 